\title{
A Plan for Internet Directory Services
}

\section{Table of Contents}

1. Introduction

1.1. The Issues

1.2. Project Summary

3. Pre-existing Services

4. Proposed Approach

4.1. Stage 1: The Field Test 12

4.2. Stage 2: Implementation 17

4.3. Stage 3: Deployment 17

5. Conclusion

Status of this Memo

This memo proposes a program to develop a directory service for the Internet. It reports the results of a meeting held in February 1989, which was convened to review requirements and options for such a service. This proposal is offered for comment, and does not represent a committed research activity of the Internet community. Activity in this area is anticipated, and comments should be provided promptly. Distribution of this memo is unlimited.

1. Introduction

1.1. The Issues

As part of the planned growth of the Internet (in particular, in support of the full science research community in the U.S.), an increasing need is anticipated for various sorts of directory services. The increase in the size of the community served by the Internet and the burgeoning demands for electronic mail lead to the need for a service to find people's computer mailboxes and other relevant facts, a so-called "White Pages" service. At the user level to date, there have been no such national or international white pages services in general use. As part of building the National Research Network (NRN), it is important that such a service exist, not only within the NRN community, but also crossing the boundaries from the NRN to the more global network community. This will enhance communication not only among computer scientists, but also among 
scientists and engineers in other fields as well. Also important and related is a so-called "Yellow Pages" service, which permits the location of Internet resources based on their attributes.

A "White Pages" service is one in which one can look up people in order to learn information about them for finding them. In its simplest form, a white pages service provides what the white pages telephone book provides. Based on a name, one can find an address and a telephone number. In a network environment, there may be many other kinds of location information, such as electronic mailbox, electronic calendar, or file server, where one might leave a file for the recipient. In addition, the electronic white pages may support a much more sophisticated set of mechanisms for lookup. One might match on a more complex set of attributes than first and last name. In addition, the searching might span more than one local white pages service. There are a number of naming and directory service specifications and implementations in the field. They have differing functionality and mechanisms to address that functionality.

Within the the world of networking today, there are a number of partial solutions to the directory service problem. Examples of these are the Internet Domain Naming Service (DNS), Clearinghouse, DECnet Network Architecture Naming Service (DNANS), Profile, and X.500. The Domain Naming Service provides a directory service most commonly used for host naming and mail delivery. Clearinghouse and DNANS are respectively the Xerox and DEC corporate naming services, originally for mail delivery, although having other uses as well, in both cases. Profile is part of the work of Larry Peterson to explore descriptive naming in a non-hierarchical structure.

There is a CCITT recommendation X.500 (ISO DIS 9594), which defines a general directory service. One of its primary goals is the naming service needed for message handling (X.400). While X.500 is still developing, and would need further evolution to cover all the requirements of a service for the Internet, it will have an important impact on the Internet community. It will form the basis of commercial products, and it will almost certainly be the directory service of many parts of the network world, which implies a need to interoperate at a minimum. There is some concern that despite the fact that X.500 is a recognized standard, there are a number of gaps and limitations of the approach, that in turn will cause it to be inadequate for the needs of the NRN.

In this context, a meeting was held to review current requirements and solutions for directory services. This RFC reports the results of that meeting, including the possibilities for a program of work in this area. 
For two days, a group representing academic, commercial, and government interests in directory services discussed both alternative candidates for a white pages service and the issues in building any such service. The meeting was kept small by inviting only a small number of representatives of each perspective. By the conclusion of the second day, a consensus was reached on how one could achieve a white pages service in three years. This is summarized in the next section.

\subsection{Project Summary}

The consensus of the meeting can be summarized in the following five points:

1. The standards and implementations are close enough to being complete that it is reasonable to undertake provision of an NRN "White Pages" service.

2. Although we are close, an effort is needed to experiment with different levels of service, to flesh out the standards, and to develop code.

3. An initial evaluation experiment is needed before making final detailed plans for a production version of the service.

4. With strong funding and encouragement, a production service is possible in three years.

5. It is important to act now to provide a coherent solution. This means both having an impact on the evolving standards and providing a unified, wide-spread solution before a plethora of differing solutions appear.

Although it has clearcut drawbacks, X.500 was identified as the most likely candidate directory service. The reasons for this are that it has rich semantics and is becoming the accepted international standard. However, there are problems with its incompleteness and with its strict hierarchy. Therefore, in order to explore these and become convinced of its viability, the consensus at the meeting was to propose field trials, as the project's first stage. The field trials would be limited in the user community, perhaps restricted to computer science departments because of their familiarity with the problems, and would be based on experimental or new software. They would include experiments with at least an X.500 implementation, Profile, and DNANS. Each of these services has strong points that must be considered as part of the evaluation. They are: 


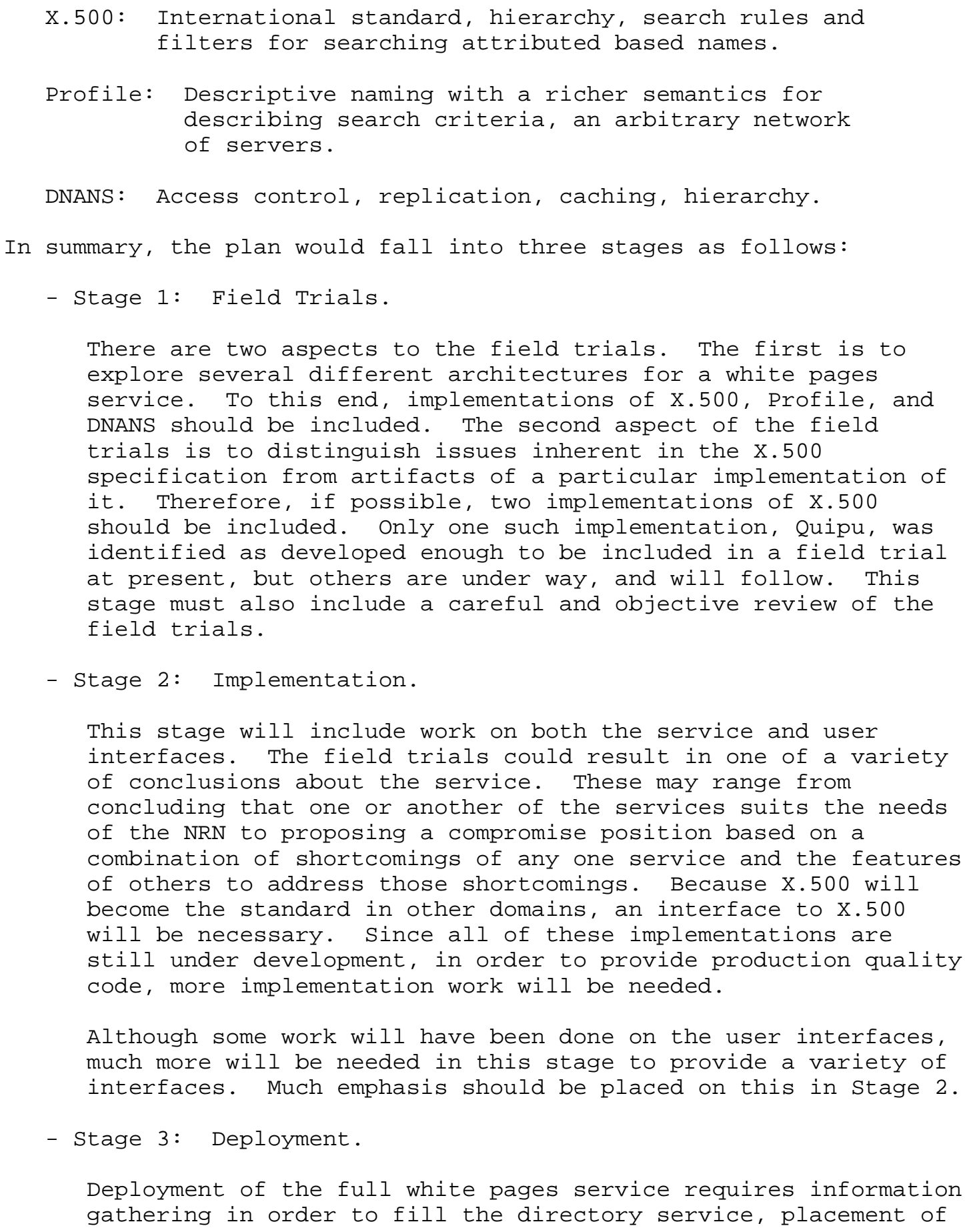


servers, distribution of and training for use of client code, placement and management of services, and delegation of authority within the service for authority over the contents. Data collection and some delegation of authority as well as training for users of the client code would begin during the field trial. This stage would begin concurrently with the other two. During the second year, detailed planning for deployment must take place. This stage would conclude in three years, at which time widespread deployment would have occurred.

In order to undertake this three stage program effectively, the group identified the following major projects:

- Further implementation of code for the field trials.

In each case (e.g., Quipu, Profile, and DNANS), programs exist, although modifications are likely to be necessary. For example, each will need to be modified to utilize the common file format into which the input data about users will be gathered.

- Design, development and evaluation of user interfaces.

- Design and development of data gathering and management tools.

- Oversight and evaluation of the field trials.

Careful thought and planning must go into the field trials, to guarantee that we learn what is needed to make an evaluation and to plan for the white pages service. The evaluation must also produce a document that is both a general specification (assuming no one alternative is chosen wholesale) and profiling information, in order for later interoperability and conformance testing.

- Detailed planning and later management of deployment.

This includes delegation of authority over parts of the namespace and arbitrating the shape of the namespace (addressing the questions about who gets what sorts of names). This is in addition to the continued and extended data collection and management, distributing the data, placing the code, documentation and user education.

- Standards participation is an important part of the program. It is critical as $\mathrm{X} .500$ changes during the next 4 year study period that the United States take a strong stand on any 
changes we envision. It is encumbant on us to utilize effectively the results of the largest field trials of this work in the international arena. The group agreed that this could take up to one half of one person's time in a year.

- A task force or working group is necessary to provide a forum for communication and discussion.

It is important to pursue this path now, both to architect a unified solution before a collection of ad hoc solutions is deployed, and to provide effective input into the X.500 standards work based on the field trials.

2. Goals and Requirements for a White Pages Service

The requirements of a white pages service are the following:

- Functionality:

The simple form of a white pages service is straightforward; one should be able to query the service with the name of a person, and have returned attributes of the person such as network mail address and phone number.

- Correctness of information:

The information in a white pages service is useless and untrusted if it is not updated regularly. A white pages service will not be used, if the information it provides is out of date or incorrect. This will require a set of management tools. Data integrity is an especially difficult challenge in this area, in contrast with information that is syntactically correct.

- Size:

The science and research community has been estimated at ten million users. The number of organizations in the United States is on the order of ten to one hundred thousand.

- Usage and query rate:

In comparison with the typical telephone book pattern of about one lookup a week per person, users of electronic mail in the science and research community will send more electronic mail messages than they currently make phone calls, leading to an estimate of ten searches a week per user for electronic as well as paper mail and telephone information. This leads to a query 
rate of $10 * \star 8$ queries per week or 170 per second on average, with much higher peak rates. The average could probably be handled by a single server, but not the peak rates and this would leave little room for growth. Therefore, a distributed, multiple server solution is the only one that make sense.

- Response time:

The issue of overall query behavior must be considered carefully. The issue arises when queries, in particular searches, are not limited to tightly constrained sets of entries. Since the number of queries generated will be proportional to the number of users (and the size of the system), the white pages design must avoid costs per query that are related to the size of the system. The consequence, otherwise, will be quadratic behavior in response time.

The response time of the service must also reflect the expected usage. A phone book style query must respond in the waiting time tolerable to a user, perhaps ten seconds maximum, or one second desirable. If the service is incorporated as a component of a larger service, then the needs of that service determine the response time.

- Partitioned Authority:

The white pages service under discussion would be used by a wide variety of organizations, ranging from small and large companies, to network service providers, to government agencies. Many of these would find it unacceptable to delegate the authority over their namespaces to some other organization. Therefore, partitioned authority including some access control, name assignment, and information management must be possible.

- Access Control:

The access control required by the white pages falls into two categories, read access control, and write or modify access control. There are at least two reasons that read access control must be available. One is that organizations may require limiting the access to the actual entries or parts of them. This would be comparable to organizations not being willing to distribute their corporate phone books or personnel records. The other reason is that some organizations do not want to publicize or make public their organizational structure. Write and modify access control is necessary because both individuals and organizations may want to prevent inadvertent or malicious creation or modification of 
information. Access control is an issue for both organizations wanting to retain local control of personnel information and individuals wanting to control access to private information about themselves.

- Multiple Transport Protocol Support:

Within the next three years, one cannot expect all the organizations in the USA to convert to the OSI protocols. On the other hand, some will. It is therefore important that any white pages service provide interfaces on top of both OSI protocols and TCP/IP. There currently exists a partial OSI suite know as ISODE on top of TCP. This is being distributed widely enough that perhaps this should also be supported.

In addition to these requirements, there are a number of features that would make a white pages service more useful. These are:

- Additional Functionality:

Descriptive naming with sophisticated searching based on attributes would support a more flexible human interface than simple name translation. Descriptive naming also would support a general yellow pages style service.

The form of a yellow pages service is less certain. One definition of a yellow pages service is a directory that stores a number of pre-computed inversions of the directory database, so that entries can be retrieved very efficiently using these predetermined attributes of the data. Another definition of a yellow pages service is one that provides a very powerful set of search primitives, somewhat in common with a relational query language, to support retrieval of entries that match complex attribute conditions. In other words, one view of a yellow pages service is that it is constructed to avoid expensive searches, the other is that it is to facilitate general searches.

- Accountability:

Accountability is important both for allocation and recovery of costs. Vendors may provide commercial directory services, therefore depending on accounting as part of their successful commercial ventures.

- Multiple Interfaces:

There should be both human and programming interfaces to the 
white pages. For example, in addition to human lookups, mail services could effectively use a naming service allow users to include human oriented names than the current electronic mail addresses that are required, such as full domain names.

- Multiple Clients:

Several different clients should exist both to provide for a variety of styles of human usage, and to support selection of the most commonly used computer environments (e.g., UNIX, VMS, MSDOS, OS2, MAC/OS).

3. Pre-existing Services

This section identifies other naming services that have been proposed or implemented for naming people. Implementations of all of these exist, although some are still only experimental.

Internet Domain Naming Service

The Internet Domain Name Service $[6,1]$ is used today to name host machines. It is implemented to address the query rates and database sizes consistent with looking up hosts as part of mail delivery. It provides a hierarchy with delegation of authority within the hierarchy. Aliases are also available. There is no access control, and the service is widely distributed throughout the Internet. It supports management of distribution, replication and caching. It is operational, and provides a rich base of practical experience. It was originally intended to be extensible to cover naming of people. It runs on a variety of different operating systems and utilizes the TCP/IP protocol suite.

The DECnet Network Architecture Naming Service (DNANS)

There is a rather well developed specification [5,3] for a naming service that is part of the DECnet architecture, which in turn arose from work at the DEC SRC in Palo Alto. This architecture addresses some problems not yet covered by X.500, such as access control, replication, and caching. It was explicitly defined to have great scalability and management features. It provides a global hierarchy of names, which are mapped into properties. Therefore, operations of searching based on properties or attributes may be expensive and difficult. At present it is only implemented on VMS using the DNA protocols, but will be moved to UNIX and TCP in the next year. 


\section{Clearinghouse}

This service [7,2] is part of the Xerox network environment. It operates today as a global service for Xerox. They have considerable experience with its operation, including problems of scale. Clearinghouse provides a three-level hierarchy of names that are mapped to sets of properties. Loose consistency is provided through slow propagation of updates. Both this service and the DEC service mentioned above are to some extent based on an earlier Xerox service called Grapevine.

Profile

A project at the University of Arizona run by Larry Peterson [8] has produced a white pages name service called Profile. It supports descriptive naming and sophisticated lookup tools. Profile assumes the existence of some other service such as the DNS to navigate among Profile servers. This navigation service need not restrict the relationship among Profile servers to a hierarchical organization; Profile supports a non-hierarchical global structure. Names in Profile consist of sets of attributes. Experimental implementations are in operation today, and the largest site currently contains about 10,000 entries. The Profile code has been available for long enough that it has become stable. The implementation is UNIX-based only and uses TCP.

$\mathrm{X} .500$

X.500 is the CCITT recommendation (also ISO/IEC/DIS 9594) [4] for a directory service. Because it is a CCITT recommendation, it evolves in four year study periods, one of which has recently come to a close. Thus, X.500 has a stable definition for the next four years.

In X.500, the set of all objects forms a single hierarchy, with each object being named relative to its parent and a single root as the topmost parent. An object consists of a set of attributes. Searching can be done by use of a logical combination of attribute values, known as a filter. A subset of these attributes comprise an object's distinguished name relative to its parent. The hierarchy as described in the CCITT recommendation is geographic at its top level and organizational within that. Alternatives can also be defined, although they are not part of the CCITT or ISO documents. In addition, there is no proposed mechanisms for distributing information about other attribute types or object classes. As with the other services, $\mathrm{X} .500$ is a distributed service. It 
specifies cooperating servers or Directory Server Agents (DSAs) under local control and management each of which knows about one or more parts of the hierarchy. The clients are known as Directory User Agents (DUAs). It is defined to run on top of the OSI protocol stack. The demonstrations of X.500 in the context of Internet run on top of the ISODE package, which provides OSI transport on top of TCP.

X.500 is incomplete in that there are a number of identifiable areas in which the standard says nothing, but that need to be specified for a successful implementation. Some examples of these are: access control (although authentication is supported), replication, caching, the database itself (the shape of the hierarchy), tools to limit the scope and cost of searching, and database management tools.

There are currently a small number of implementations of X.500 in progress at such locations as University College London (the Quipu project, on UNIX using ISODE), the University of British Columbia (UNIX based using their own full OSI suite), MIT (experimental, Symbolics Lisp Machine based, Lisp using TCP), The Wollongong Group (offshoot of Quipu), The Retix Corporation, NIST, and at least several underway in Italy and Japan. There are probably others and a number of other American corporations have discussed building their own. Each of these must make its own decision in the areas in which X.500 is silent. Quipu is probably the most complete implementation of X.500 to date. The pilot version has about 20 DUAs in seven countries with an estimated 20,000 entries total.

\section{Proposed Approach}

The conclusion of this report is that some form of $\mathrm{x} .500$ is the most likely candidate. The reasons for this decision are that it has a rich semantics and will become the international de facto standard. There are, however, serious problems with its incompleteness and with its strict hierarchy. Therefore, in order to explore these and become convinced of its viability, the attendees at the meeting agreed on field trials, as a first stage. Initially, this would include experiments with at least one X.500 implementation (Quipu), Profile to explore a non-hierarchical structure and richer descriptive naming, and DNANS in order to explore some of the incomplete aspects of X.500 for which DNANS has architected solutions.

A three-stage plan, with all three stages beginning coincidentally and as soon as possible, would provide such a service within the NRN. The first stage should be complete in a year, the second in two, and 
the third in three. Stage 1 would be field trials of three approaches to naming with an emphasis on distinguishing between the specification and a particular implementation of X.500, as well. Stage 2 would be a more complete implementation of a white pages service base on the conclusions from Stage 1 . Stage 3 would be widespread deployment of the implementation developed in stage 2 . The planning for stage 3 is not outlined here in detail, because that plan would be part of the proposed work to be done. If the field trials were to lead to the conclusion that none of the services is adequate, the plan for the remainder of the work would need to be rescheduled.

If the Internet community is to adopt X.500 (or any other standard), it is necessary to make a number of design and management decisions, above and beyond the implementation decisions for the DSA. Since there are a number of such decisions to be resolved, and some of these are significant, the group recommended that this planning and management function should be recognized as a distinct activity.

\subsection{Stage 1: The Field Test}

It was agreed that field trials would be a valuable form in which to explore the issues of building a white pages service for two reasons. First, the software is still in early stages of development or deployment. Some of it is production code, but still first release; the rest is part of research projects. Second, it is important to learn from experience with a limited and sympathetic community. The suggested community was the computer science community, in particular, computer science departments. That will not be the case completely, since the computer science community in general does not use DECnet. Therefore, for experiments with the DNANS, the NASA/DOE community was recommended. They will be using DNANS in any case, as they move to DECnet Phase V.

The twofold purpose of the field trials is to explore differing directory service architectures and to refine the study of X.500 specifically, to distinguish architectural aspects of it from features of a particular implementation of x.500. Initially, the trials would include the Quipu implementation of X.500, Profile, and the DNANS. A second implementation of X.500 should be identified and included as soon as possible. Part of the emphasis of the field trials would be on gathering and maintenance of naming information. To ease this, a single common file format for storage of and access to the naming information and use of a single set of data management tools was recommended, although no particular set was identified. The various directory services would need to be retrofitted to this file format. Such consistency in file format would mean that the services could all be co-resident, sharing files, thus permitting 
single locations to participate in several parts of the field trials. This, in turn, would allow for direct comparisons.

There are a number of issues, which are not addressed in X.500, that would need to be resolved for a large scale deployment such as a white pages for the NRN. In particular, these are: clients of the service; data collection and maintenance; distribution, replication and caching of information; access control, accountability, and information integrity; and support by non-OSI protocols. Each of the name services included in the field trials would include decisions in these areas, albeit different ones. The field trials will allow for evaluation of these different mechanisms.

There are two other major issues that must also be addressed: functionality and size. Functionality encompasses both the first point of the nature of the interfaces to the service as well as the structure of the namespace (e.g., hierarchy). A discussion of size must include not only the number of entries handled by the service as a whole, but how those entries are distributed and the query and update patterns.

In general, all of these issues are tightly coupled, but are separated here for the purposes of understanding the field trials and its potential effectiveness. They would also be the issues that would be the basis for the work done in stage 2 of the project.

- Functionality:

X.500 and DNANS make strong statements about the organization of the namespace. In both cases, it is a single, absolute hierarchy with soft links or aliases and attribute-based naming useful both in searches of subtrees of the hierarchy and for storing information about the objects in the hierarchy. The searches are based on logical combinations of attribute values. Quipu implements the naming structure and search functionality as specified in X.500. In contrast, Profile, provides a more general facility that supports any form of relative names, not just hierarchical, and a small programming language to express the functions for searching. By including Profile in the field trials, these more general facilities can be tested.

X.500 specifies that the service is separated into two parts for implementation of the service, known as the Directory Service Agent (DSA), and the client, known as the Directory User Agent (DUA). DUAs can be implemented independently of the implementation of the white pages service. Quipu, Profile, and DNANS have taken different approaches to the presentation model for DUAs, so the three implementations will allow for 
additional experience.

- Size:

As discussed earlier, a white pages service must be prepared to handle a minimum of $10 * \star 7$ entries, although they may be distributed, and a query rate of hundreds per second. It must also be prepared to handle much higher peak rates. If the address lookup that is presently provided by the DNS is also supported by the white pages service, the query rate will be much higher. The designers of the field trials must determine whether or not such usage will be part of the final service and therefore must be examined in the field trials. If so, caching may be part of the solution. In addition, the response time for DUAs must be reasonable for a human sitting at a console. Furthermore, modifications to the data should occur in reasonably short periods of time, although this could be measured in hours.

The field trials must allow for experimentation under such stressful conditions. The environment for testing must have both large and small nodes, as well as both heavy and light load querying and situations in which reorganization can be tested. Such reorganization may be a simple as moving one piece of the hierarchy to another point and handling naming conflicts in the new environment. X.500 does not address this issue, but it will be needed by the NRN.

- Distribution, replication, and caching:

These are areas in which $\mathrm{X} .500$ has very little to say, but a great deal of work has been done in other distributed, network naming services, in particular both the DNS and DNANS. There seems to be general agreement that distribution of naming services should be done on the basis of nodes in the naming structure, which also provide the basis for administrative partitioning. All the naming services described here support distribution, partitioning of the information for placement on cooperating servers. Neither X.500 (and therefore Quipu) nor Profile is prepared to redistribute portions of the namespace, for reallocation of administrative responsibilities or load balancing, although this should be possible and DNANS is prepared to do so. Replication is necessary for accessibility in a large-scale or global namespace, although again X.500 does not address this issue. Quipu has taken a stand on this, by defining master and slave copies of the data; it is similar to, but not the same as, the approach taken in the DNS. Caching is barely touched on in X.500 and not at all in Profile, but our 
experience with the DNS indicates that caching is critical to effective operation of a distributed name service. The DNANS has an architected solution based on objects in the namespace as the unit of distribution and replication. Again, the DNANS solution should be explored in the field test environment.

- Access control, accountability, and integrity:

Access control and accountability require some degree of authentication. X.500 supports authentication based on using an RSA public key algorithm, but does not address issues of universal registration, nor issues of access control or accountability themselves. These are left as a local issue, although depending on the design of the system, they may have global implications. The problem of integrity of the information in the name service is nowhere addressed. Profile also does not address these issues, although it uses authentication based on UNIX authentication, involving user ids and passwords. DNANS takes a strong stand on access control, architecting it in at the level of individual entries. Field trials will force these issues out into the open.

- Structure of the naming tree:

In the deployment of the DNS, about one year was lost to arguments about the actual structure of the naming hierarchy. People form strong opinions about their name, and fight for or against certain hierarchical structures. The same issue will arise here, and advanced planning to deal with the problem is required.

In this case, the problem is made harder by the fact that the hierarchy will be global; X.500 is an international standard, based on the assumption that there is only one example of the tree, partitioned by country. Probably the American White Pages Service, at least at its root, will be run by the NIST or its contractor. We must deal with the problem that in the short term, various implementations may not interwork, and we must work with NIST to support the needed services.

Specific issues that come up related to the naming tree are:

* How is delegation of control of the tree managed? For example, who decides what DSA holds what parts of the tree?

* How is the creation of new parts of the tree (e.g., an organizational entry) controlled? 
- Support for Tree Search:

Regardless of the defintion of the white pages service in the NRN, it will need to interface to the $\mathrm{x} .500$ world. The $\mathrm{x} .500$ naming hierarchy can be expected to become very large, and guidance is needed for users to help them navigate the tree. Users need help to find their way to unknown parts of the namespace. As in other naming services, a feature of $\mathrm{X} .500$ is that additional entries, aliases (similar to links in file systems) can be installed to provide an easy path for a user in one part of the tree to find other interesting parts of the tree. By establishing a consistent policy for the use of alias entries, learning how to navigate the tree can be made much easier for a user. As part of setting up the tree, therefore, these sorts of policies need to be defined.

- Definition of database structures:

There are a number of data structures that need to be defined as part of setting up each of the services. These include, for example, the types of information stored for the entry about a person. This information must be stored in the servers, and passed to the clients. These structures must thus be specified. In other words, the schema defining attributes and object classes must be specified for the NRN.

- Load balancing:

The dynamic performance of the Internet system must be estimated, so that the servers can be sized properly. Especially at the root of the tree, the query rate must be estimated carefully. Caching will have a strong influence on this. Therefore, traffic patterns are very dependent on the details of implementation.

- Supporting multiple protocol suites:

At least three protocol suites are and will continue to be used in the NRN environment. They are DECnet, TCP/IP, and the OSI suite of protocols. Since the white pages service is at the applications layer, it must run on top of at least these three protocol suites. It is important to understand the requirements of the white pages service for its transport protocols.

By addressing these issues within the field trials, we will be preparing for the further development of Stage 2. A result of Stage 1 will be a detailed specification of the white pages service, 
possibly an extension to or modification of X.500. This should dovetail with the activities specifying the details required for implementation (known as "profiling") by the NIST Workshop for Implementors of OSI. In addition, in order to run the field trial, the information capture problem must be addressed, providing the some of the preliminary work of stage 3 .

\subsection{Stage 2: Implementation}

If the evaluation of Stage 1 concludes that some form of $\mathrm{X} .500$ is acceptable, at least one of the two X.500 implementations included in the field trials should provide the basis for a production quality implementation of X.500 for general deployment. Further work will likely be needed on the basis of the evaluations of the field trials. A production version of an implementation requires both reliable servers as well as a variety of clients to provide differing interfaces on a mixture of hardware and operating systems.

In addition, especially because of the inclusion of Profile and DNANS, a variety of different DUAs will be explored by definition. Further investigation into the DUAs should begin in parallel with or in conjunction with the field trials. There should be distinct DUAs for both programs and humans. In addition, there probably should be human-user DUAs geared both to the naive user with simple usage patterns and the more sophisticated user who wants to perform complex queries. It is also important to design DUAs that do not require a great deal of computing power for the small machines still in use in great quantity. Much of the user community may not be able to afford expensive equipment upgrades.

Assuming that $X .500$ is deemed to be the specification of the service, the field trials will address many issues not included in X.500 as of 1989. Since it is important for the NRN to support interconnectivity beyond its own bounds, it behooves us to feed what has been learned back into the standards activities. This was identified as a separate activity because of the intellectual as well as time commitment that must be made to do this effectively.

\subsection{Stage 3: Deployment}

A plan is required to develop the schedule of service introduction, and to co-ordinate the deployment as it is undertaken. This includes mediating service problems, a significant task in its own right.

The details of deployment were not discussed at the meeting, although several of the seeds of deployment lie in stages 1 and 2 . The first of these is the capture and management of information. The second is DUA development. Both of these must be included stage 1 in order to 
support a usable environment for the trials. In addition, the information that will have been captured in stage 1 could be printed producing a hard copy of the white pages information. That could be distributed to all scientists and engineers involved; such a project would provide an early white pages service. During the initial periods of both stages 1 and 2, planning for deployment would also have to proceed, in order to provide a smooth transition to this third stage in the project.

5. Conclusion

The consensus of the meeting was that following a path that included X.500 was both the correct direction and feasible, although X.500 needs further elaboration. There were several important items for further study. The first is that there are many issues left unresolved in X.500 that have been addressed in other naming services, and the NRN should take advantage of the solutions in those other services. The second is that there was some reservation about certain features of X.500, especially in the area of the imposition of a hierarchy for naming, and only limited flexibility in descriptive naming. The participants believe that is important understand whether X.500 provides enough mechanisms to work around such problems by finding a higher common ground that includes the best features of all the naming services included in the field trials. The final issue with respect to $\mathrm{X} .500$ was that there was agreement that X.500 will be an accepted and utilized standard in at least part of the networked community and therefore interfacing to it will be necessary. Given that, and the other reasons for choosing $\mathrm{X} .500$, the consensus was that the plan described above would bring the NRN and its community of users a useful and usable white pages service.

References

1. Austein, R., "The Internet Domain Name System", Proceedings of USA Decus, Massachusetts Institute Technology/LCS, 1987.

2. Demers, A., D. Greene, C. Hauser, W. Irish, J. Larson, S. Shenker, H. Sturgis, D. Swinehart, and D. Terry, "Epidemic algorithms for replicated database maintenance", Proceedings of the 6th Symposium on Principles of Distributed Computing, ACM, Vancouver, B.C., Canada, pp. 12-21, August 1987.

3. Digital Equipment Corporation, "DNA Naming Service Functional Specification Version 1.0.1", Order number: EK-DNANS-FS-001, Digital Equipment Corporation, 1988.

4. International Organization for Standardization, "Information 
Processing Systems - Open Systems Interconnection - The

Directory", Draft Standard (In 8 parts), Also CCITT

Recommendation X.500, 1988 .

5. Lampson, B., "Desiging a Global Name Service," Proceedings of the 5th Symposium on Principles of Distribute Computing, ACM, Calgary, Alberta, Canada, pp. 1-10, August 1986.

6. Mockapetris, P., "Domain Names - Concept and Facilities", RFC 1034, USC/Information Sciences Institute, November 1987.

7. Oppen, D., and Y. Dalal, "The Clearinghouse: A Decentralized Agent for Locating Named Objects in a Distributed Environment", Tech. Rept. OPD-T8103, Xerox Corporation, Palo Alto, CA, October 1981 .

8. Peterson, L., "Profile: A System for Naming Internet Resources", Tech. Rept. 20, Department of Computer Science, University of Arizona, June 1987.

Author's Address

Karen R. Sollins

Massachusetts Institute of Technology

Laboratory for Computer Science

545 Technology Square

Cambridge, MA 02139-1986

Phone: (617) 253-6006

EMail: SOLLINS@XX.LCS.MIT.EDU 